\title{
Expression of Dikaryon-specific and Non-specific mRNAs of Schizophyllum commune in Relation to Environmental Conditions and Fruiting
}

\author{
By J. G. H. WESSELS, * G. H. MULDER AND J. SPRINGER \\ Department of Plant Physiology, Biological Centre, University of Groningen, Kerklaan 30 , \\ 9751 NN Haren, The Netherlands
}

(Received 11 November 1986; revised 20 February 1987)

\begin{abstract}
cDNA clones representing eight mRNAs expressed abundantly in the dikaryon of Schizophyllum commune at the time of fruiting, but very low in the progenitor monokaryons, were used to estimate the concentrations of these mRNAs in cultures of the dikaryon and monokaryon growing under conditions that suppress the formation of fruit-bodies. Such conditions as high atmospheric carbon dioxide and continuous darkness in surface cultures or growth in suspension cultures generally lower the concentrations of these mRNAs in the dikaryon but do not bring them down to the very low levels of these mRNAs in the monokaryons. Therefore the expression of these mRNAs, which depends on the dikaryotic condition, may be required but is not sufficient for the occurrence of fruiting.
\end{abstract}

\section{INTRODUCTION}

When two coisogenic monokaryons of Schizophyllum commune differing only in the alleles of the two incompatibility genes $A$ and $B$ are mated, a dikaryon arises which is genetically identical to the progenitor monokaryons except for the presence of two different alleles of each of the incompatibility genes. Phenotypically this results in a marked change in hyphal morphology in which mononucleate cells are converted into binucleate cells with clamp connections at all division septa (Raper, 1983). In addition, unlike the monokaryons, the dikaryon readily forms fruit-bodies under proper environmental conditions. This process is accompanied by the appearance of a limited number of abundantly expressed mRNAs which are at a much lower concentration in the young vegetatively growing dikaryon and even lower or undetectable in the monokaryons at all growth stages (Mulder \& Wessels, 1986; Wessels, 1985).

Enhanced atmospheric carbon dioxide and continuous darkness suppress fruit-body development in $S$. commune (Raudaskoski \& Salonen, 1984). Fruit-body formation is also absent in shaken suspension cultures. Here we show that such conditions lower but do not completely suppress the appearance of the dikaryon-specific mRNAs.

\section{METHODS}

Organism and culture conditions. The coisogenic monokaryon strains of Schizophyllum commune 4-39 (A4IB41, CBS 341.81) and 4-40 ( $A 43 B 43$, CBS 340.81) were mated to produce the dikaryon 4-39/4-40. The strain 4-40 belonged to a series of strains which was regularly backcrossed to strain 4-39 to maintain isogenicity. The monokaryons and the dikaryon were grown from mycelial homogenates. For surface-grown mycelia the homogenate was evenly spread over a $0.7 \%(\mathrm{w} / \mathrm{v})$ agar medium as described previously (Dons et al., 1979; Hoge et $a l ., 1982 b)$. The medium $(300 \mathrm{ml})$ was contained in Fernbach flasks $(2000 \mathrm{ml})$ which were continuously flushed with humidified air $\left(5-81 \mathrm{~h}^{-1}\right)$ either supplemented or not with $5 \%(\mathrm{v} / \mathrm{v})$ carbon dioxide. Flasks receiving carbon dioxide were incubated in the light (fluorescent tubes, about $1000 \mathrm{~lx}$ ) and flasks receiving air were incubated either in the light or in complete darkness. Incubations were at $24^{\circ} \mathrm{C}$ and cultures were harvested $4 \mathrm{~d}$ after inoculation. This was done by separating mycelial mats from the semisolid medium with a spatula after which the mats with adhering agar medium were frozen in liquid nitrogen and stored at $-70^{\circ} \mathrm{C}$. For suspension-grown mycelia, the mycelial homogenate was inoculated into $250 \mathrm{ml}$ Erlenmeyer flasks containing $100 \mathrm{ml}$ liquid minimal medium. 
The flasks were incubated on a gyrorotary shaker (180 r.p.m.) at $24^{\circ} \mathrm{C}$ for various periods. The mycelia were harvested by centrifugation, frozen in liquid nitrogen and stored at $-70^{\circ} \mathrm{C}$. Glucose concentrations in the medium were measured by the glucose oxidase method (GOD-PAP, Boehringer Mannheim).

Isolation of total RNA. Frozen mycelium was ground to a powder under liquid nitrogen in a mortar and the RNA was extracted by a small-scale procedure based on the hot-phenol extraction procedure described earlier (Hoge $e t$ al., 1982a). Results of denaturing gel electrophoresis indicated intact RNA.

Estimation of the concentration of specific mRNAs. Glyoxalated samples of total RNA (1-3 $\mu \mathrm{g})$ were dotted onto Genescreen-plus (NEN) and hybridized to $\left[\alpha^{-32} \mathrm{P}\right] \mathrm{dCTP}$-labelled molecular probes as described previously (Mulder \& Wessels, 1986). Hybridization signals were quantified by scintillation counting. The probes used were cDNA sequences originally cloned in pBR327 and representing mRNAs specifically expressed in the fruiting dikaryon (pSc-1, 2, 4, 5, 6, 7, 9 and 14) or mRNAs present in both monokaryotic and dikaryotic mycelia (pSc-3, 10 and 11). Isolation and some characteristics of these clones have been described (Mulder \& Wessels, 1986). For the experiments described here the cDNA inserts were actually recloned into pGEM-1 vectors (Promega Biotec). The mRNA and the gene corresponding to pSc-1 have been completely sequenced (Dons et al., 1984), but for the other DNA clones only the insert lengths and the lengths of the corresponding mRNAs are available (Table 1). Various concentrations of unlabelled pSc-1 insert, mixed with yeast RNA, were also dotted onto membranes to serve as a standard for converting hybridization signals into absolute concentrations of the probed mRNAs. Concentrations are given as percentages of total RNA in the culture. Assuming RNA/DNA ratios of 73 and 47 for monokaryon and dikaryon, respectively (de Vries \& Reddingius, 1984), an amount of DNA per nucleus of $0.04 \mathrm{pg}$ at G1 (Dons et al., 1979), and $58 \times 10^{-8} \mathrm{pg}$ for the weight of an average mRNA molecule, the number of copies of an mRNA molecule per cell can be estimated. An mRNA molecule at a concentration of $1 \times 10^{-3} \%$ of total RNA would then be present with an average of 50 copies in monokaryotic cells and of 65 copies in dikaryotic cells.

\section{RESULTS}

\section{Surface cultures}

Surface cultivation of the $S$. commune dikaryon in a stream of air in the light gave rise to the development of numerous cup-shaped fruit-bodies (ca. $50 \mathrm{~cm}^{-2}$ ) between the third and fourth day. The monokaryon remained completely vegetative. The dikaryon-specific mRNAs reached high concentrations in the fruiting dikaryon but were not detected in the monokaryons with the exception of a low concentration of Sc-4 mRNA in monokaryon 4-39 (Table 1). The concentrations of various dikaryon-specific mRNAs in the fruiting dikaryon resembled those found previously under slightly different growth conditions at the same time of harvesting

Table 1. Abundance of dikaryon-specific and non-specific mRNAs in a dikaryon and a monokaryon of Schizophyllum commune after $4 d$ in surface culture under conditions affecting fruiting in the dikaryon

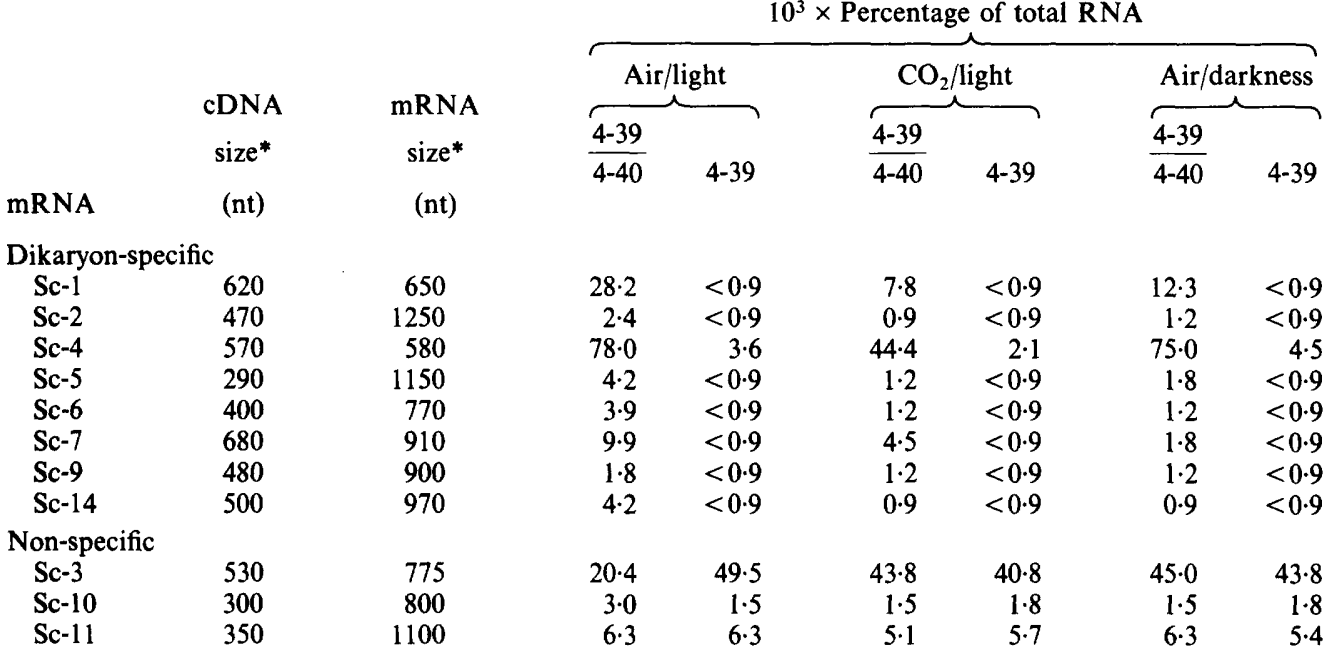

* From Mulder \& Wessels (1986); nt, no. of nucleotides. 


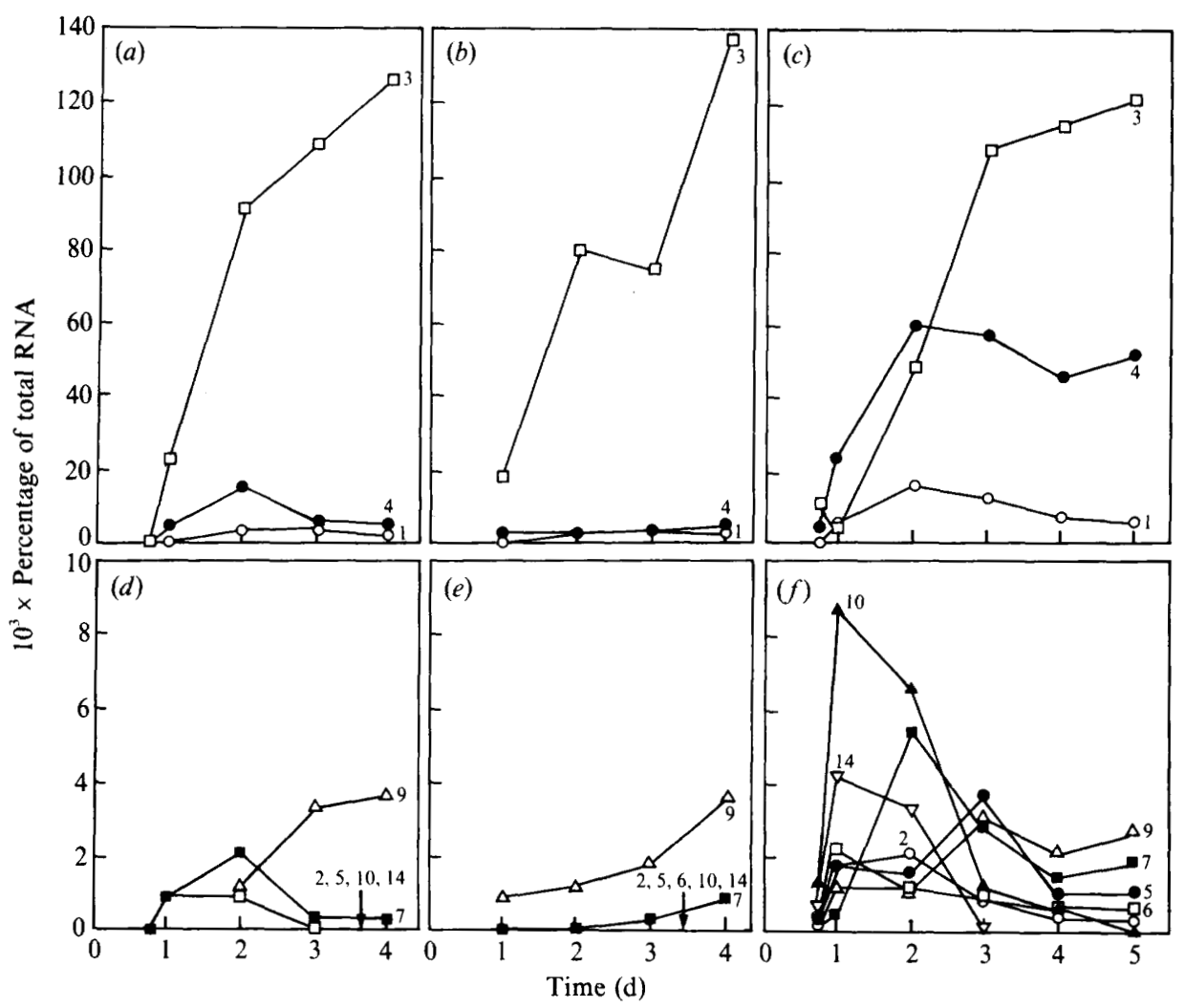

Fig. 1. Temporal expression of cloned RNA sequences in shaken suspension cultures of monokaryon strains 4-39 $(a, d)$ and 4-40 $(b, e)$ and the derived dikaryon 4-39/4-40 $(c, f) .(a, b, c): \bigcirc, \mathrm{Sc}-1 ; O, \mathrm{Sc}-4 ; \square$, Sc-3. $(d, e, f): O$, Sc-2;, Sc-5; $\square$, Sc-6;, Sc-7; $\triangle$, Sc-9; $\Delta$, Sc-10, $\nabla$, Sc-14. mRNAs not shown for the monokaryons were too low to be detected.

(Mulder \& Wessels, 1986). Small deviations probably arose from the fact that the mRNAs were changing concentrations rapidly at this time while, in addition, these aerated cultures lagged somewhat in development compared to the non-aerated cultures used before. The non-specific mRNAs were present in both the dikaryon and the monokaryon (Table 1), but note that the Sc-3 mRNA was at a higher concentration in the monokaryon.

As expected, cultivation of the dikaryon in a stream of $5 \%(\mathrm{v} / \mathrm{v})$ carbon dioxide in the light or in a stream of air in complete darkness resulted in abolition of fruit-body development. The dikaryon and the monokaryon now looked similar macroscopically although, of course, the difference in hyphal morphology persisted. These environmental conditions also generally suppressed the levels of the dikaryon-specific mRNAs (Table 1). However, the suppression was only partial and the level of the Sc- 4 mRNA was hardly affected by cultivation in darkness. The imposed environmental conditions had little effect on the levels of the non-specific mRNAs; their concentrations became similar in monokaryon and dikaryon under fruit-body-inhibiting conditions. Only the Sc-3 mRNA in the dikaryon was clearly elevated by these conditions.

\section{Suspension cultures}

As in surface cultures, the monokaryons and the dikaryon grew at the same rate in shaken suspension cultures with about $75 \%$ of the glucose consumed at the fourth day in all cases. In suspension culture the dikaryon could not form fruit-bodies and the only visible difference between the monokaryons and the dikaryon concerned the hyphal morphology. Fig. 1 shows the changes in abundance of the various mRNAs during growth in suspension culture. All values 
were derived from one experiment except for the values given for $18 \mathrm{~h}$ of cultivation, which were derived from step-up cultures (Zantinge et al., 1979) in order to obtain enough young mycelium with a high incidence of apical cells. The concentrations of the dikaryon-specific mRNAs, except the Sc-9 mRNA, remained low in the monokaryons but increased in the dikaryon after the early phases of growth. However, the maximum values remained somewhat lower than those reached in the fruiting surface cultures (Table 1). In a parallel 4-d-old surface culture of the dikaryon the following abundances were found $\left(10^{3} \times \%\right.$ of total RNA in parenthesis): Sc-1 (33.3), Sc-2 (2.1), Sc-4 (103.8), Sc-5 (11.1), Sc-6 (10.2), Sc-7 (15.9), Sc-9 (4.8) and Sc-14 (0.9). Of the non-specific sequences, the Sc-10 mRNA was not detectable in the monokaryons but reached rather high values in the dikaryon. The non-specific Sc-3 mRNA behaved similarly in monokaryons and the dikaryon, being very low at the early phases of growth but reaching extremely high concentrations (around 7500 copies per cell) in older cultures. In the parallel 4-d surface culture of the dikaryon it amounted to $13.5 \times 10^{-3} \%$ of total RNA (around 900 copies per cell).

\section{DISCUSSION}

Previously (Mulder \& Wessels, 1986), a relationship between the dikaryon-specific mRNAs and fruiting was surmised because $(i)$ these $\mathrm{mRNAs}$ were found in suspension-grown dikaryon at levels less than $10 \%$ of those found in surface-grown fruiting dikaryon, (ii) they increased steeply in concentration concomitant with fruit-body formation, (iii) they were present in higher concentration in the fruit-body initials than in the supporting mycelium, and (iv) they were also prevalent in full-grown fruit-bodies. It now appears that the first argument is not entirely correct because the dikaryon-specific mRNAs did appear in suspension cultures when cultivation was continued beyond $1 \mathrm{~d}$ (Fig. 1), although at concentrations less than those found in fruiting surface cultures (Table 1). However, the low concentration of these mRNAs in the young suspension-grown dikaryon and the absence of clear differences with the monokaryons at this stage (Fig. 1) still argue against a role of these mRNAs in dikaryon morphogenesis as occurring in apical cells. We estimate that in cultures less than $1 \mathrm{~d}$ old $20-30 \%$ of the hyphal compartments consist of such apical cells. Indeed, preliminary experiments (unpublished) indicate that in colonies of the dikaryon growing on agar, these mRNAs are only abundantly present at some distance from the periphery of the colonies in the area where also the fruit-bodies arise.

At first sight, the suppression of the levels of the dikaryon-specific mRNAs by environmental conditions that prevent fruit-body formation would seem to indicate a causal relationship. However, it is equally possible that the imposed environmental conditions indirectly lower the concentration of the dikaryon-specific mRNAs by preventing fruit-body formation in some unknown way. These mRNAs are most abundant in the fruit-bodies but are also present in lower concentration in the supporting vegetative mycelium (Mulder \& Wessels, 1986). We have estimated that in the profusely fruiting dikaryon up to $80 \%$ of the RNA in the culture may be present in the fruit-bodies. In cultures in which fruit-body formation is prevented only vegetative mycelium is formed and the abundance of the dikaryon-specific mRNAs in the whole culture may thus drop to the lower levels found in the supporting vegetative mycelium of fruiting cultures. In this interpretation there would be no direct regulation of the level of the dikaryonspecific mRNAs by the environmental factors and the lower levels found in non-fruiting cultures would be a consequence of the absence of fruit-bodies. Such an interpretation would also accord with the observation (Table 1, Fig. 1) that the non-specific Sc-3 mRNA in the dikaryon increased to levels found in the monokaryon under conditions imposing vegetative growth on the dikaryon, because this mRNA is present at much lower concentration in the fruitbodies than in the supporting vegetative mycelium (Mulder \& Wessels, 1986). In any case, this study suggests that if the dikaryon-specific mRNAs are required for fruit-body development then their abundant appearance is not initiated by the fruiting process nor are they sufficient for fruiting to occur.

Experiments are under way to localize precisely the dikaryon-specific mRNAs in the differentiating mycelium by in situ hybridizations and to probe the occurrence of these mRNAs 
during monokaryotic fruiting. Replacement of the normal genes by in vitro-mutagenized copies of the isolated genes would provide the best test for function. The recent development of a transformation system for $S$. commune (Munoz-Rivas et al., 1986) and the demonstration of gene replacement by transformation in another filamentous fugus (Miller et al., 1985) indicate the feasibility of such experiments.

This work was supported by the Foundation for Fundamental Biological Research (BION), which is subsidized by the Netherlands Organization for the Advancement of Pure Research (ZWO).

\section{REFERENCES}

Dons, J. J. M., DE VRIES, O. M. H. \& Wessels, J. G. H. (1979). Characterization of the genome of the basidiomycete Schizophyllum commune. Biochimica et biophysica acta 563, 100-112.

Dons, J. J. M., Mulder, G. H., Rouwendal, G. J. A., Springer, J., Bremer, W. \& Wessels, J. G. H. (1984). Sequence analysis of a split gene involved in fruiting from the fungus Schizophyllum commune. EMBO Journal 3, 2101-2106.

Hoge, J. H. C., Springer, J., Zantinge, B. \& WESSELS, J. G. H. (1982a). Absence of differences in polysomal RNAs from vegetative monokaryotic and dikaryotic cells of the fungus Schizophyllum commune. Experimental Mycology 6, 225-232.

hoge, J. H. C., Springer, J. \& Wessels, J. G. H. $(1982 b)$. Changes in complex RNA during fruitbody initiation in the fungus Schizophyllum commune. Experimental Mycology 6, 233-243.

Miller, B. L., Miller, K. Y. \& Timberlake, W. E. (1985). Direct and indirect gene replacements in Aspergillus nidulans. Molecular and Cellular Biology 5, 1714-1721.

Mulder, G. H. \& Wessels, J. G. H. (1986). Molecular cloning of RNAs differentially expressed in monokaryons and dikaryons of Schizophyllum commune in relation to fruiting. Experimental Mycology 10, 214227.

Munoz-Rivas, A., Specht, C. A., Drummond, B. J., Froeliger, E., Novotny, C. P. \& Ullrich, R. C.
(1986). Transformation of the basidiomycete Schizophyllum commune. Molecular \& General Genetics 205, 103-106.

RAPER, C. A. (1983). Controls for development and differentiation in basidiomycetes. In Secondary Metabolism and Differentiation, pp. 195-238. Edited by J. M. Bennett \& A. Ciegler. New York: Marcel Dekker.

Raudaskoski, M. \& Salonen, M. (1984). Interrelationship between vegetative development and basidiocarp initiation. In The Ecology and Physiology of the Fungal Mycelium, pp. 291-322. Edited by D. H. Jennings \& A. D. M. Rayner. Cambridge: Cambridge University Press.

DE VRIES, O. M. H. \& ReddingIUS, J. (1984). Synthesis of macromolecules and compartment size in monokaryotic and dikaryotic hyphae of Schizophyllum commune. Experimental Mycology 8, 378-381.

WESSELS, J. G. H. (1985). Gene expression during basidiocarp formation in Schizophyllum commune. In Molecular Genetics of Filamentous Fungi, pp. 193206. Edited by W. E. Timberlake. New York: Alan R. Liss.

Zantinge, B., Dons, J. J. M \& Wessels, J. G. H. (1979). Comparison of poly(A)-containing RNAs in different cell types of the lower eukaryote Schizophyllum commune. European Journal of Biochemistry 113, $251-260$. 